\title{
TECNOLOGIAS DE ENFERMAGEM NO MANEJO DA DOR EM RECÉM-NASCIDOS NA UNIDADE DE TERAPIA INTENSIVA NEONATAL
}

Amanda Santana de Medeiros Nóbrega ${ }^{1}$, Anajás da Silva Cardoso Cantalice ${ }^{2}$, Ana Carolina Dantas Rocha Cerqueira ${ }^{3}$, Nathanielly Cristina Carvalho de Brito Santos, Nycarla de Araújo Bezerra ${ }^{3}$, Thayane Ramires Santos Chaves ${ }^{3}$

Objetivo: verificar tecnologias de enfermagem utilizadas no manejo da dor em recém-nascidos de uma Unidade de Terapia Intensiva Neonatal. Metodologia: pesquisa quantitativa, descritiva, exploratória, realizada em uma Maternidade Pública Estadual no interior da Paraíba, com 12 técnicos de enfermagem e 5 enfermeiros. Utilizado um questionário estruturado para coleta de dados e a análise feita por meio do Microsoft Excel. Resultados: foi observado que a utilização de escalas de dor não faz parte da rotina e o choro foi o parâmetro mais utilizado para reconhecer a dor no neonato. Conclusão: é necessário ampliar as discussões sobre essa temática a partir da sistematização e educação permanente nos serviços de cuidados intensivos ao neonato.

Descritores: Unidades de Terapia Intensiva Neonatal, Manejo da dor, Enfermagem neonatal, Tecnologia biomédica.

\section{NURSING TECHNOLOGIES IN THE MANAGEMENT OF PAIN IN NEWBORNS IN A NEONATAL INTENSIVE CARE UNIT}

Objective: verifying the nursing technologies used in the management of pain in newborns in a Neonatal Intensive Care Unit. Methodology: this is a descriptive exploratory quantitative research, performed in a Public Maternity placed in interior of Paraiba's State, with 12 nursing technicians and 5 nurses, a structured questionnaire was used for the collection of data the analysis made through the Microsoft Excel. Results: it was noticed that the use of pain scales is not part of the routine and the crying was the most used parameter by the nursing team to recognize pain in the neonate. Conclusion: it is necessary to broaden the discussions on this subject from the systematization and continuous education in the neonatal intensive care services.

Descriptors: Neonatal Intensive Care Units, Pain Management Neonatal Nursing, Biomedical Technology.

\section{TECNOLOGÍAS DE ENFERMERÍA EN EL MANEJO DEL DOLOR EN RECIÉN NACIDOS EN LA UNIDAD DE CUIDADOS INTENSIVOS NEONATALES}

Objetivo: verificar tecnologías de enfermería utilizadas en el control del dolor en recién nacidos de una unidad de cuidados intensivos neonatales. Metodología: pesquisa cuantitativa, descriptiva y exploratória, realizada en una Maternidad Pública Estatal en el interior de la Paraiba, con 12 tecnicos de enfermería y 5 enfermeros. Fue utilizado un cuestionario estructurado para la recogida de datos y la análisis hecha por medio del Microsoft Excel. Resultados: se observó que el uso de escalas de dolor no era la rutina y el llanto fue el parámetro mas usado para reconocer el dolor de neonato. Conclusión: es necesario aumentar las discusiones sobre ese tema a partir de la sistematización y educación permamente en los servicios de cuidados intensivos al neonato.

Descriptores: Unidades de Cuidados Intensivos Neonatales, Manejo del dolor, Enfermería neonatal, Tecnología biomédica.

IUniversidade Federal de Campina Grande (UFCG). E-mail: amanda__nobrega@hotmail.com.

${ }^{2}$ Universidade Federal de Campina Grande (UFCG) e da Faculdade de Ciências Médicas de Campina Grande.

${ }^{3}$ Universidade Federal de Campina Grande (UFCG). 


\section{INTRODUÇÂO}

No Brasil, tem se observado, nos últimos dez anos, um aumento da prematuridade. Dados do Sistema de Informações de Nascidos Vivos (SINASC) apontam a intensificação de nascimentos pré-termos na maior parte das capitais. Considera-se recém-nascido pré-termo (RNPT) todo aquele nascido com menos de 37 semanas e a termo aquele cuja idade gestacional é de 37 a 41 semanas e 6 dias².

Ao longo da internação do recém-nascido (RN) em uma Unidade de Terapia Intensiva Neonatal (UTIN), a dor é um sintoma contínuo, referente aos quadros clínicos graves e os inúmeros procedimentos invasivos. Um recém-nascido em uma UTIN recebe cerca de 130 a 234 manipulações nas 24 horas, sendo a maioria bastante dolorosa ${ }^{2}$. Ador é determinada pela Sociedade Internacional para o Estudo da Dor (IASP) como uma experiência sensitiva emocional desagradável relacionada à lesão real ou potencial dos tecidos, tratandose de uma manifestação abstrata, envolvendo mecanismos físicos, psíquicos e culturais ${ }^{3}$.

A equipe de enfermagem desempenha papel fundamental no controle da dor e na redução do sofrimento do recémnascido, permanecendo junto ao doente grande parte do tempo de internação, além de ser diretamente responsável por procedimentos invasivos que são bastante frequentes em ambientes de unidades de cuidados neonatais ${ }^{4}$. Portanto, é necessário desconstruir o tradicional argumento de que o RN não tem capacidade de sentir dor, dado que os elementos funcionais e neuroquímicos do sistema nervoso são essenciais para que ocorra a transmissão do impulso doloroso para o córtex cerebral do neonato, fazendo com que o RN sinta dor (5).

Nesse cenário, énecessário avaliaras tecnologias utilizadas no manejo da dor no neonato internado em unidade de terapia intensiva neonatal, submetido à diversos procedimentos invasivos, pelo fato da dor neonatal ser interpretada como fator prejudicial para o desenvolvimento adequado do $\mathrm{RN}^{4}$. Portanto, o presente estudo buscou investigar as tecnologias de enfermagem utilizadas no manejo da dor em recémnascidos em uma Unidade de Terapia Intensiva Neonatal.

\section{METODOLOGIA}

Pesquisa quantitativa, descritiva e exploratória que buscou verificar as tecnologias de enfermagem utilizadas no manejo da dor em recém-nascidos, realizada em uma Maternidade Pública Estadual no interior da Paraiba. É um hospital de referência para a realização de partos de alto risco, que dispõe de seis leitos de UTI Obstétrica e 10 leitos de UTI Neonatal. A população do estudo foi constituída pela equipe de enfermagem da Unidade de Terapia Intensiva Neonatal, composta por 36 participantes, sendo 28 técnicos de enfermagem e 8 enfermeiros. A pesquisa ocorreu no periodo de junho a julho de 2016.

O instrumento de coleta de dados consistiu em um questionário estruturado desenvolvido pelo pesquisador, que contemplou questões relativas a variáveis sociodemográficas e profissionais, dados referentes à identificação e alivio da dor, quanto à conduta profissional diante da dor do recémnascido, e, como esse profissional fazia uso das tecnologias de enfermagem no cuidado ao RN.

A análise dos dados ocorreu por métodos estatísticos, por meio do Microsoft Excel, possibilitando a tabulação e organização dos dados para posterior análise.

O estudo obedeceu à Resolução 466/12 do Conselho Nacional de Saúde do Ministério da Saúde, conforme o parecer de número 1.721 .463 , sendo garantida a privacidade dos participantes da pesquisa por meio da assinatura do Termo de Consentimento Livre e Esclarecido (TCLE) por parte do profissional de enfermagem legal.

\section{RESULTADOS}

A equipe de enfermagem que atua em uma Maternidade Pública Estadual no interior da Paraiba é composta por 36 profissionais, sendo 8 enfermeiros e 28 técnicos de enfermagem. Desses, dois enfermeiros não quiseram participar da pesquisa, uma enfermeira estava de férias, enquanto que entre os técnicos, 12 não quiseram participar da pesquisa, dois estavam em período de vacância, um técnico de férias e um estava de licença. Totalizaram assim 17 participantes, sendo cinco enfermeiros e 12 técnicos de enfermagem. (Tabela 01).

Tabela 01 - Caracterização dos profissionais de enfermagem da UTIN. Paraiba, Brasil.

\begin{tabular}{|lcc|}
\hline Caracteristicas & Enfermeiros $(\mathbf{n})$ & Técnicos de Enf. $(\mathbf{n})$ \\
\hline Feminino & 4 & 12 \\
\hline Masuclino & 1 & - \\
\hline Faixa etária & \\
\hline 30-39 anos & 2 & 3 \\
\hline $40-49$ anos & 1 & 5 \\
\hline mais de 50 anos & 1 & 2 \\
\hline União estável & 1 & 2 \\
\hline Solteiro & Estado Civil & 4 \\
\hline Casado & 2 & 5 \\
\hline
\end{tabular}




\begin{tabular}{|c|c|c|}
\hline Viúvo & - & 1 \\
\hline Divorciado & - & 1 \\
\hline Possui Latu sensu & 5 & - \\
\hline Possui strictu sensu & 1 & - \\
\hline Participação em cursos & 3 & 9 \\
\hline \multicolumn{3}{|c|}{ Experiência profissional } \\
\hline menos de 1 ano & - & - \\
\hline $1-5$ anos & 2 & 4 \\
\hline 6-10 anos & 1 & 1 \\
\hline mais de 10 anos & 2 & 7 \\
\hline \multicolumn{3}{|c|}{ Experiência em UTIN } \\
\hline menos de $l$ ano & 1 & - \\
\hline $1-5$ anos & 2 & 5 \\
\hline 6-10 anos & 2 & 3 \\
\hline mais de 10 anos & - & 4 \\
\hline
\end{tabular}

Ao serem questionados sobre a avaliação da dor no RN e sua frequência, todos os entrevistados afirmaram que avaliam a dor, sendo que quatro dos enfermeiros avaliam sempre, e seis técnicos avaliam frequentemente e cinco avaliam sempre.

No que diz respeito ao uso de escalas para avaliação da dor no neonato, os profissionais de enfermagem responderam não utilizá-las, alegando que seu uso não fazia parte da rotina da UTIN onde foi realizada a pesquisa. O choro foi considerado o sinal de existência de dor mais evidente por toda a equipe de enfermagem, seguido da mímica facial.

Quando questionados sobre a importância do tratamento da dor no RN, todos os profissionais de enfermagem relataram sua importância e, todos os enfermeiros, declararam que tratar a dor do RN na UTIN melhora o prognóstico. Por outro lado, entre os técnicos de enfermagem onze afirmaram que o tratamento da dor no RN diminui seu sofrimento (Tabela 2).

Tabela 02 - Distribuição do uso das ferramentas utilizadas pelos profissionais de enfermagem na identificação da dor no recém-nascido e achados sobre a importância do seu tratamento. Paraiba, Brasil.

\begin{tabular}{|c|c|c|}
\hline Variáveis & Enfermeiros (n) & Técnicos de Enf. (n) \\
\hline \multicolumn{3}{|c|}{ Escala de dor } \\
\hline Sim & - & - \\
\hline Não & 5 & 12 \\
\hline \multicolumn{3}{|c|}{ Mimica facial } \\
\hline Sim & 5 & 10 \\
\hline Não & - & 2 \\
\hline
\end{tabular}

\begin{tabular}{|lcc|}
\hline & Choro & \\
\hline Sim & 5 & 12 \\
\hline Não & - & 2 \\
\hline & Movimentos & \\
\hline Sim & 4 & 8 \\
\hline Não & 1 & 4 \\
\hline & Parâmetros Fisiológicos & \\
\hline Sim & 2 & 2 \\
\hline Não & 3 & 10 \\
\hline & Melhora o prognóstico & 5 \\
\hline Sim & 5 & 7 \\
\hline Não & - & 11 \\
\hline & Diminui o sofrimento & 4 \\
\hline Sim & 4 & \\
\hline
\end{tabular}

Os profissionais participantes foram questionados também se conhecem alguma medida não farmacológica para o alivio da dor em neonatos, e todos afirmaram não só conhecer, como executá-las para minimizar/reduzir esta dor diante de procedimentos ou condições dolorosas diversas. As medidas não farmacológicas citadas pelas duas categorias da enfermagem, com respectivas frequências relativa e absoluta, encontram-se listadas na Tabela 3.

Tabela 03 - Medidas terapêuticas ou preventivas utilizadas pela equipe de enfermagem para o alivio da dor no RN. Paraíba, Brasil.Sim

\begin{tabular}{|lcc|}
\hline & Variáveis & Técnicos de Enf. (n) \\
\hline Sim & Escala de dor & - \\
\hline Não & - & 12 \\
\hline & 5 & \\
\hline Sim & Mimica facial & 10 \\
\hline Não & 5 & 2 \\
\hline & - & \\
\hline Sim & Choro & 12 \\
\hline Não & 5 & - \\
\hline & - & 8 \\
\hline Sim & Parâmetros Fisiológicos & 4 \\
\hline Não & 2 & 2 \\
\hline & Movimentos & \\
\hline
\end{tabular}




\begin{tabular}{lcc} 
Não & 3 & 10 \\
\hline Sim & Melhora o prognóstico & \\
\hline Não & 5 & 5 \\
\hline Sim & - & 7 \\
\hline DISCUSSÃO & Diminui o sofrimento & 11 \\
\hline
\end{tabular}

Sabendo então de alguns possiveis fatores que podem levar o recém-nascido a se caracterizar como de risco, destaca-se que, na vida intrauterina, o RN passa por sequenciais transformações decisivas para o seu crescimento e desenvolvimento e, caso após o nascimento, o processo adaptativo ao meio extrauterino não seja eficaz, algumas crianças podem necessitar de acompanhamentos e cuidados complexos, frequentemente realizado na Unidade de Terapia Intensiva Neonatal ${ }^{6}$.

No presente estudo, foi observada uma maioria de profissionais do sexo feminino, com uma média de idade de aproximadamente 38 anos. Estudo similar, realizado em uma maternidade pública de Fortaleza com 25 técnicos de enfermagem, também encontrou a totalidade das técnicas de enfermagem de uma UTIN como sendo do sexo feminino e com média de idade semelhante. Esses dados evidenciam a construção histórica da enfermagem, em que o cuidar é associado ao sexo feminino. A média de idade mais elevada pode ter relação com o maior grau de experiência requerido na UTIN?.

Foi verificado, ainda, que tanto os enfermeiros como os técnicos de enfermagem tinham uma média de 10 anos de experiência profissional. Nesse contexto, a implementação da neonatologia é atual na maioria das instituições de saúde do interior do Brasil, pois era restrita aos grandes centros urbanos. No entanto, com a proposta de descentralização da saúde, o investimento governamental tanto em estrutura física, recursos tecnológicos e no fortalecimento dos hospitais, tem conquistado espaço nas instituições hospitalares do interior do Brasil ${ }^{8}$.

Quando se interrogou a equipe de enfermagem se concordavam se o recém-nascido sente dor, todos confirmaram que os RNs sentem dor, visto que há evidências de sua capacidade neurológica para captar a dor. As ferramentas utilizadas pelos profissionais de enfermagem na identificação da dor neonatal citados no questionário foram a utilização de escalas de dor, mimica facial, choro, movimentos corporais e parâmetros fisiológicos. Percebeu-se que a utilização de escalas de dor não faz parte da rotina da instituição onde foi realizada a pesquisa e alguns profissionais relataram desconhecer as escalas de mensuração da dor. $O$ choro foi o parâmetro mais utilizado pela equipe de enfermagem, sendo citada por todos os profissionais de enfermagem seguido da mimica facial.

É necessária a sistematização no processo de avaliação e tratamento da dorno RN pela enfermagem, pautada em ações, atitudes e intencionalidades da equipe de enfermagem, tendo como base o conhecimento científico, experiência, intuição e pensamento crítico, com o intuito de promover, manter e recuperar a saúde do neonato(9). Um estudo realizado em Sergipe, com 90 pacientes, mostrou que, apesar da dor ser considerada o quinto sinal vital, sua classificação é feita de forma inadequada, associada ao tratamento fora do padrão da escala de verificação da dor, sendo pouco explorado e com poucos registros ${ }^{7}$.

O choro foi um parâmetro unânime indicado pela equipe de enfermagem da UTIN onde foi realizada a pesquisa, sendo um sinal considerado mais importante para avaliar a dor no neonato. A utilização da avaliação do choro de modo isolado, na prática, é muito questionável, pois o choro pode ser desencadeado por outros estímulos que não sejam o doloroso, como desconforto, frio, fome, posição desconfortável, sono, além de que neonatos farmacologicamente debilitados e entubados, são incapazes de emitir o choro $^{10}$.

Quanto ao uso de medidas não farmacológicas para o alivio da dor em recém-nascidos, todos os profissionais de enfermagem afirmaram ter conhecimento sobre essas medidas como também, de fazerem seu uso para minimizar/ reduzir a dor diante os eventos dolorosos. As medidas utilizadas foram: chupeta de gaze com glicose, acalento, pacotinho, sucção não-nutritiva, método mãe-canguru, shantala e balneoterapia.

O método mãe-canguru (MMC) foi a medida nãofarmacológica mais utilizada pelos enfermeiros e o pacotinho mais utilizada pelos técnicos de enfermagem. O uso de medidas não farmacológicas tem se destacado como estratégias terapêuticas a fim de minimizar os danos causados no RN. Elas objetivam, principalmente, prevenir o processo doloroso, a desorganização do neonato, o estresse e a agitação. São eficientes quando utilizadas individualmente nas dores de leve intensidade $e^{4,11}$.

O MMC é um método que traz inúmeros benefícios, melhorando significativamente o quadro do neonato, pois promove o aleitamento materno, minimiza o estresse, propiciando um melhor desenvolvimento físico e emocional do $\mathrm{RN}$ e diminuído os riscos causados pelos procedimentos a que são submetidos(12). O pacotinho se refere ao posicionamento do RN de forma mais confortável possivel, como um embrulho. Tal posição faz com que o bebê se sinta protegido durante o procedimento, proporcionando aconchego ${ }^{5}$.

No que diz respeito a chupeta de gaze com glicose, 
também muito citada pela equipe de enfermagem na instituição onde foi realizada a pesquisa, a utilização dessa medida causa liberação de opióides endógenos, os quais possuem características analgésicas intrínsecas, bloqueando os caminhos da dor, diminuindo o tempo do choro, a frequência cardiaca e os escores das escalas de dor, devendo ser administrada, em média, dois minutos antes da realização de procedimentos dolorosos ${ }^{13}$

A shantala e balneoterapia são pouco utilizados pela equipe de enfermagem e, quando questionados sobre a balneoterapia, a maioria dos técnicos de enfermagem desconheciam tal prática. Na balneoterapia ou o banho terapêutico, o RN é imerso no balde de ofurô, deixando-o mais tranquilo, e estimulando a sução, diminuindo a dor neonatal. Quanto a shantala, é uma massagem que estimula os sistemas musculoesquelético, gastrointestinal, nervoso, circulatório e linfático, promovendo o relaxamento, melhorando a qualidade do sono e, minimizando os efeitos dolorosos decorrentes dos procedimentos a que o neonato é submetido na UTIN ${ }^{14,15}$.

Como pode ser visto, a equipe de enfermagem da instituição onde foi realizada a pesquisa, reconhece e coloca em prática o uso de medidas não-farmacológicas, porém é notório que é realizado de forma não sistematizada. O RN permanece em um ambiente onde o uso de aparatos tecnológicos são necessários para assegurar a vida desse ser, o que torna a UTIN um ambiente hostil e frio, sendo imprescindivel reduzir o impacto da tecnologia dando lugar ao cuidado humanizado, buscando acolher e respeitar os valores, as crenças, culturas, realizando um cuidado pautado na necessidade individualizada e a resposta de cada neonato/ familia frente ao cuidado humanizado, sendo importante o enfermeiro conhecer a comunicação verbal e não verbal emitida pelos bebês e o familiar durante o desenvolvimento do cuidado (9,16). A qualificação da equipe é ferramenta fundamental para que o nível de cuidado humanizado descrito seja alcançado e as consequências ao RN implicadas pela internação sejam mínimas.

Como limitação do estudo, destacou-se baixo quantitativo detécnicos de enfermagem que responderam ao questionário, porém não dificultou os resultados desse estudo, que foram de grande importância e incentivo para novas pesquisas voltada para essa temática.

\section{CONCLUSÃO}

O profissional de enfermagem é o principal personagem no cuidado direto ao recém-nascido na UTIN, ficando claro que o seu papel é o de ininterruptamente minimizar/evitar o evento doloroso causado pelos diversos procedimentos invasivos a que o RN é submetido.

Foi observado que, entre as tecnologias de enfermagem para o manejo da dor mais utilizadas pela equipe de enfermagem, se destacaram: método mãe-canguru, pacotinho e acalento. Além disso, pode se evidenciar, ainda, a não adesão por partes dos profissionais do uso das escalas de avaliação da dor neonatal e a utilização do choro como um dos parâmetros mais importantes na avaliação da dor neonatal.

Existe uma necessidade dessas tecnologias serem realizados de forma sistematizada e continuamente, sendo, portanto, necessária a amplificação dessas discussões e educação permanente nos serviços de cuidados intensivos ao RN. 


\section{REFERÊNCIAS}

1. Balbino AC, Cardoso MVLML, Silva RCC, Moraes KM. Recém-nascido pré-termo: respostas comportamentais ao manuseio da equipe de enfermagem. Rev Enferm. 2012 dez; 20(esp.1): 615-620. Disponivel em: http://www.epublicacoes.uerj.br/index.php/enfermagemuerj/article/ view/5908

2.Cruz CT, Stumm EMF. Instrumentalização e implantação de escala para avaliação da dor em Unidade de Terapia Intensiva Neonatal. Relato de caso. Rev dor. 2015 set; 16(3): 232-234. Disponivel em: http:// dx.doi.org/10.5935/1806-0013.20150046

3.Alves FB, Fialho FD, Dias IMAV, Amorim TM, Salvador M. Dor neonatal: a percepção da equipe de enfermagem da unidade de terapia intensiva neonatal. Rev Cuid. 2013 ago; 4(1): 510-515. Disponivel em: http://www.redalyc.org/articulo.oa?id=359533224011

4.Caetano EA, Lemos NRF, Cordeiro SM, Pereira FMV, Moreira DS, Buchhorn SMM. O recém-nascido com dor: atuação da equipe de enfermagem. Esc. Anna Nery. 2013 jul-set; 17(3): 439-445. Disponivel em: http://www.scielo.br/scielo.php?script=sci_arttext\&pi$d=S 1414-81452013000300439$

5.Amaral JB, Resende TA, Contim D, Barichello E. Equipe de enfermagem diante da dor do recém-nascido pré-termo. Esc. Anna Nery. 2014 ago; 18(2): 241-246. Disponivel em: http://dx.doi.org/10.5935/14148145.201440035

6.Martins SW, Dias FS, Enumo SRF, Paula KMP. Avaliação e controle da dor por enfermeiras de uma unidade de terapia intensiva neonatal. Rev Dor. 2013; 14(1): 21-26. Disponivel em: http://dx.doi.org/10.1590/ S1806-00132013000100006

7.Mendes LC, Fontenele FC, Dodt RCM, Almeida LS, Cardoso MVLML, Silva CBG. A dor no recém-nascido na unidade de terapia intensiva neonatal. Rev enferm UFPE. 2013 nov; 7(11):6446-6454. Disponivel em: http://dx.doi.org/10.5205/reuol.3794323221ED.0711201318

8.Araújo GC, Miranda JOF, Santos DV. Camargo CL, Sobrinho CLN, Rosa DOS. Dor em recém-nascidos: identificação, avaliação e intervenções. Rev Baiana Enferm. 2015 jul-set; 29(3): 261-270. Disponível em: http://dx.doi.org/10.18471/rbe.v29i3.13695

9.Neto JAS; Rodrigue BMRD. A ação intencional da equipe de enfermagem ao cuidar do RN na UTI neonatal. Cienc Cuid Saude. 2015 jul- -set; 14(3):1237-1244. Disponivel em: http://dx.doi.org/10.4025/cienccuidsaude.v14i3.22320

10.Rosário SSD, Fernandes APNL, Araújo CSC, Paiva WWM, Batista FWB, Monteiro AI. Assistência de enfermagem ao recém-nascido com dor em uma unidade de terapia intensiva neonatal. Rev enferm UFPE. 2014 jul; Recife, 8(1): 2382-2389. Disponivel em: http://dx.doi. org/10.5205/reuol.3794-32322-1-ED.0711201318

11.Morais APS, Façanha SMA, Rabelo SN, Silva AVS, Queiroz MVO, Chaves EMC. Medidas não farmacológicas no manejo da dor em recém-nascido: cuidado de enfermagem. Rev Rene. 2016 mai-jun; 17(3): 435442. Disponivel em: http://dx.doi.org/10.5935/14148145.20140035

12.Nunes NP, Pessoa UML, Mont'Alverne DGB, Elpidio SF, Carvalho EM. Método Canguru: percepção materna acerca da vivência na unidade de terapia intensiva neonatal. Rev Bras Promoç Saúde. 2015 jul-set; 28(3): 387-393. Disponivel em: http://dx.doi.org/10.5020/18061230.2015. p387

13. Motta GCP, Cunha MLC. Prevenção e manejo não farmacológico da dor no recém-nascido. Rev Bras Enferm. 2015 jan-fev; 68(1): 131-5. Disponivel em: http://dx.doi.org/10.1590/0034-7167.2015680118p

14.Andrade MT. O banho humanizado na abordagem ao recém-nascido. Salvador. Monografia [Especialização em Fisioterapia Neonatal e Pediátrica] - Atualiza Cursos; 2014

15.Almeida AP. A massagem terapêutica em ambiente de unidade de terapia intensiva neonatal: uma revisão integrativa. Rio de Janeiro. Dissertação [Mestrado em Pesquisa Aplicada à Saúde da Criança e da Mulher] - Instituto Nacional de Saúde da Mulher, da Criança e do Adolescente Fernandes Figueira; 2014.

16.Lins RNP, Collet N, Vaz EMC, Reichert APS. Percepção da equipe de enfermagem acerca da humanização do cuidado na UTI neonatal. Rev Bras Cienc Saude. 2013; 17(3): 225-232. Disponivel em: http://dx.doi. org/10.4034/rbcs.2013.17.03.03 